\title{
$\underline{\mathbf{P}-97}$
}

\section{Potential of Syzygium Malaccense as Antimicrobial Preservative Agent for Topical Application Products}

\author{
Norulaiman Yusoff*, Ong Boo Kean, Saidatul Husni Saidin, Saiful Azmi Johari, Nor Azah \\ Mohamad Ali and Mohd Irman Shah Mustafar
}

Herbal Product Development Programme, Natural Products Division, Forest Research Institute Malaysia FRIM, 52109

Kepong, Selangor, Malaysia; E-mail: norulaiman@frim.gov.my

\begin{abstract}
Antimicrobial preservatives are substances that are added into formulation or product to prevent microbial spoilage and thus, prolong the product shelf-life. Our preliminary study indicated ethanolic extract of Syzygium malaccense leaf (SmL) exhibited antimicrobial activity via the minimum inhibitory concentration (MIC) and minimum bactericidal concentration (MBC) test. $\mathrm{SmL}$ was then incorporated into a formulated topical application product before being subjected to a preservative efficacy test. Microbial preservative efficacy of $\mathrm{SmL}$ at $0.25 \%(\mathrm{w} / \mathrm{v})$ and $0.5 \%(\mathrm{w} / \mathrm{v})$ were evaluated against Staphylococcus aureus, Pseudomonas aeruginosa, Escherichia coli, and Candida albicans for the duration of 28 days. Euxyl-K300 (0.5\%, w/v)), a synthetic chemical preservative was used as positive control in the study. The preservative efficacy study showed that both the $0.25 \%(\mathrm{w} / \mathrm{v})$ and $0.5 \%(\mathrm{w} / \mathrm{v})$ of SmL exhibited inhibitory activity against $S$. aureus on the 7 day and against C. albicans on the 21 day. The SmL has the potential to be developed as antimicrobial preservative agent in the topical application products.
\end{abstract}

Keywords: Syzygium malaccense, preservative efficacy, topical application. 